\title{
Editorial: Re-valorization of Food Losses and Food Co-products
}

\author{
Jesus Simal-Gandara ${ }^{1}$, Tripti Agarwal ${ }^{2}$, Mahnaz Esteki $^{3}$, Andrea Gomez-Zavaglia ${ }^{4 *}$ and \\ Jianbo Xiao ${ }^{1}$ \\ ${ }^{1}$ Nutrition and Bromatology Group, Department of Analytical Chemistry and Food Science, Faculty of Food Science and \\ Technology, University of Vigo, Vigo, Spain, ${ }^{2}$ Department of Agriculture and Environmental Sciences, National Institute of \\ Food Technology Entrepreneurship and Management, Sonipat, India, ${ }^{3}$ Department of Chemistry, University of Zanjan, Zanjan, \\ Iran, ${ }^{4}$ Center for Research and Development in Food Cryotechnology (CIDCA-CONICET La Plata), La Plata, Argentina
}

Keywords: food loss and food waste, agriculture, nutraceuticals, functional foods, food recovery hierarchy

\section{Editorial on the Research Topic}

\section{Re-valorization of Food Losses and Food Co-products}

According to the report on the World State of Food and Agriculture 2019, progress in the fight against food loss and waste, and the reduction of food loss, help to improve the sustainability of the environment, while the reduction of waste benefits food security. According to the FAO, the Food and Agriculture Organization of the United Nations, global food waste represents a third of total food produced for consumption, $\sim 1.6$ billion tons per year, which represents a cost of $€$ 730 million/year. The food groups that stand out for their highest percentages of losses are fruits and vegetables, and roots and tubers, both with a $45 \%$ loss. It is followed by fish and marine products with $35 \%$, and cereals with $30 \%$. Both food losses and its co-products are very rich sources of nutrients and other bioactive substances that, once extracted, represent very valuable ingredients for the elaboration of functional and nutraceutical foods (among other products). The extraction and isolation of these compounds allow for their use in other sectors (yogurts and dairy products, juices and beverages, energy bars, etc.), as ingredients that favor certain functions when ingesting them.

The European Parliament's Waste Framework Directive applies a standard prioritization scheme for the recovery of food by-products. In it, the first option is the prevention and reduction of the generation of by-products, and the second is human consumption. Finally, there is the shipment of the by-products to landfill, a solution that cannot be considered as recovery. The continuous development of re-valorization solutions will accelerate the sustainability of the food production and consumption system. This Research Topic highlights the role of integrated teams of research scientists for this purpose. Following the United States Environmental Protection Agency (USEPA) food recovery hierarchy (Figure 1), we invited manuscripts that focus on solutions for revalorization of food losses and food coproducts, including but not limited to: Prevention and reduction; Human consumption; Bioproducts; Animal feeds; Industrial uses; Energy production; Agronomic uses; and Other ways of disposal.

In total, 5 articles were reviewed and published from authors mainly from America, Asia and Europe. Their topics were mainly about plant foods, but also seafood. In this way, they were centered in the production of food allergy modulators (Bessa et al.), nutraceuticals (Cortés-Ferré et al.), and animal feeds (Kithama et al.), together with approaches on green and sustainable methods (Doria et al.) and also bioconversion and bio-refinery (Venugopal). 


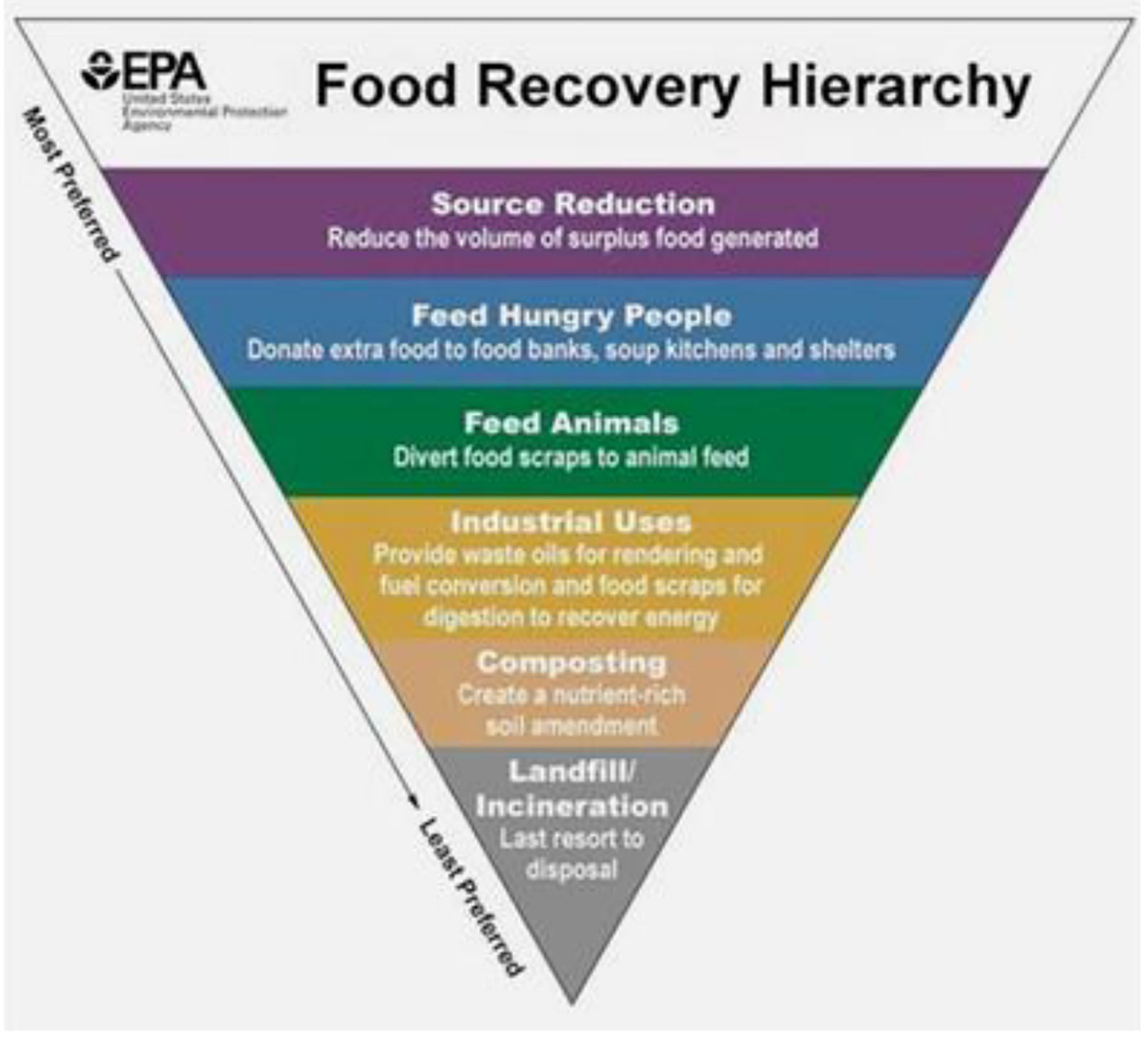

FIGURE 1 | United States Environmental Protection Agency (US-EPA) food recovery hierarchy.

\section{AUTHOR CONTRIBUTIONS}

JS-G wrote the first draft. TA, ME, AG-Z, and JX edited it. All authors approved the final version of the manuscript.

Conflict of Interest: The authors declare that the research was conducted in the absence of any commercial or financial relationships that could be construed as a potential conflict of interest.

Publisher's Note: All claims expressed in this article are solely those of the authors and do not necessarily represent those of their affiliated organizations, or those of the publisher, the editors and the reviewers. Any product that may be evaluated in this article, or claim that may be made by its manufacturer, is not guaranteed or endorsed by the publisher.

Copyright (c) 2021 Simal-Gandara, Agarwal, Esteki, Gomez-Zavaglia and Xiao. This is an open-access article distributed under the terms of the Creative Commons Attribution License (CC BY). The use, distribution or reproduction in other forums is permitted, provided the original author(s) and the copyright owner(s) are credited and that the original publication in this journal is cited, in accordance with accepted academic practice. No use, distribution or reproduction is permitted which does not comply with these terms. 